\title{
VIKOR Model of TBM Construction Risk Prediction for a Long Inclined Shaft in a Coal Mine based on Trapezoidal Vague Set
}

\author{
Yue Yang ${ }^{1,2}$, Xiaoguo Chen ${ }^{3, *}$, Honghui Huang ${ }^{4}$ and Lubo Yang ${ }^{5}$ \\ ${ }^{1}$ School of Architecture and Civil Engineering, Sanming University, Sanming 365004, China \\ ${ }^{2}$ Key Laboratory of Engineering Material and Structure Reinforement (Sanming University), Sanming 365004, China \\ ${ }^{3}$ School of Information Engineering, Sanming University, Sanming 365004, China \\ ${ }^{4}$ College of Science, Heilongjiang University of Science and Technology, Harbin 150022, China \\ ${ }^{5}$ Auburn University, Auburn, 36849, USA
}

Received 28 June 2020; Accepted 3 September 2020

\begin{abstract}
Accurate warning is realized in this study in order to improve the reliability of the construction risk analysis model of shield machine (tunnel boring machine, TBM) in a long inclined shaft of coal mine. First, the risk factors of TBM construction of a long inclined shaft in coal mine were identified, the index system of secondary risk assessment was established, and the classification standard of risk grade was determined. Then, on the basis of introducing two trapezoidal vague number algorithms, the trapezoidal vague number BM operator and the weighted arithmetic average operator were proposed, respectively, and several conclusions were obtained. The weight vector of the risk index was determined by the deviation maximization method, after which the VlseKriterijumska Optimizacija I Kompromisno Resenje (VIKOR) model based on the trapezoidal Vague number was established. Finally, the TBM construction risks of the long inclined shaft (No. 2 test well) in the Taigmiao mining area of Shenhua Group of China was evaluated, and the trends were forecasted by using this model. Results show that: (1) The risk method proposed in this study can effectively depict and deal with uncertain monitoring information. (2) Compared with the results of the scoring method and the utility value method, the rankings of the optimal and the worst risk grades are maintained, and the ranking results obtained by the scoring method and the VIKOR method are also the same. (3) The calculated monitoring results are consistent with the actual safety conditions of the coal mine construction. Conclusions indicate that the model and method are effective and practical in coal mine risk assessment, which can provide a new way for the analysis and prediction of TBM construction risks in a long inclined shaft.
\end{abstract}

Keywords: Inclined shaft TBM construction, Risk analysis, Trapezoidal vague number, Aggregation operator, VIKOR decision method

\section{Introduction}

Tunnel boring machine (TBM) construction is the most advanced tunneling method in the world and is generally prioritized as the main mode of construction when it comes to tunnel excavation. Compared with the traditional drilling and blasting method, TBM has the advantages of high efficiency, high quality and safety, and rapid speed, with a driving speed that is $4-10$ times that of the traditional drilling and blasting method. With the continuous improvement of the global coal mining technology, the adoption of inclined shaft lifting has great advantages under the conditions of medium and shallow burial depth. As the drilling and blasting method cannot significantly improve its construction speed in a short period of time, an increasing number of construction units have begun using TBM in the long-distance inclined shaft construction of coal mine under appropriate conditions. However, due to the poor adaptability of TBM to adverse engineering-geological conditions, the high requirements for the quality of construction personnel, and the complicated geological conditions of long inclined shaft construction in a coal mine, many risk factors and uncertainties are present in TBM

*E-mail address: 2321120911@qq.com

ISSN: 1791-2377 @ 2020 School of Science, IHU. All rights reserved. doi:10.25103/jestr.136.19 construction. Without effective risk management and control, this situation can lead to a variety of problems and even engineering accidents. Therefore, analyzing and evaluating the TBM construction risks of a long inclined shaft in a coal mine is of great theoretical significance and practical value.

Two aspects affect the TBM construction risks of a long inclined shaft in a coal mine. One set of problems includes the design of a risk assessment index system given that the existing studies have rarely included impact ground pressure factors, rock burst factors, harmful gas poisoning and other factors into the index system.

Therefore, building a more comprehensive evaluation index system is a prerequisite for improving the reliability of risk prediction. The second set of problems has to do with the choice of risk forecasting methods. At present, many scholars have investigated risk assessment and prediction using various classic methods, such as the analytic hierarchy process (AHP) [1], the fault tree analysis [2], fuzzy mathematics [3], Bayesian networks [4], the Grey System theory [5], the Markov chain [6], Chaos Prediction [7], the information diffusion model [8], and a variety of artificial intelligence (AI) algorithms (e.g., BP artificial neural network model [9], support vector machine (SVM) model [10], adaptive filtering algorithm [11], etc.). The AHP, Bayesian Networks, the Grey System theory, and Chaos Prediction are often used to represent the evaluation information using definite numbers; hence, they cannot 
process any uncertain evaluation information. Meanwhile, AI algorithms often cannot find the global optimal solution. The fuzzy prediction method can only depict the degree of affirmation and negation in uncertain evaluation information. They cannot determine the degree of hesitation in information, that is, the description of uncertain evaluation information is not accurate enough, and the direct conversion of fuzzy numbers into exact numbers will lead to missing additional information missing, which can affect the rationality and accuracy of evaluation results. Therefore, in order to reasonably and effectively solve the uncertainty problem in the safety evaluation of TBM construction in a long inclined shaft, a coal mine construction risk prediction method must be proposed from the perspective of a trapezoidal vague set. The relevant conclusions can provide the basis for the establishments of a safety management decision system for long inclined shafts in a coal mine.

\section{State of the art}

The proper management of security risks is important. Mollon et al. [12] analyzed the risks of tunnel engineering and established an uncertainty assessment model, thereby introducing the concept of risk management into the field of a tunnel and underground engineering for the first time. Subsequently, the risk assessment method in tunnel and underground engineering has developed rapidly. For example, Bajeel et al. [13] applied probability and statistical methods to risk analysis and established a risk decisionmaking and analysis system to evaluate tunnel risks and reliability. Aladejare et al. [14] identified the risk types of the Copenhagen subway project, proposed measures to deal with the risks, and adopted the Monte Carlo method to establish a specific risk model.

In view of the risk analysis and evaluation of water inrush in a lava tunnel, Wang et al. [15] proposed a risk evaluation method for the interval of water inrush in a lava tunnel, quantified the interval membership function and the weight of each factor, and then analyzed the comparative advantage of the interval matrix to determine the risk level. Martin et al. [16] expressed the values of influencing factors by using intuitionistic fuzzy numbers, which ensured the integrity of information representation and improved the prediction accuracy. Meanwhile, Yang et al. [17] used geographic information system (GIS) and fuzzy set theory to establish an entropy weight water inrush risk prediction model. The above three methods provide a practical reference for risk assessment and management of lava tunnel construction and have certain reference significance for the risk control of other engineering construction projects. With the aim of achieving the risk analysis and evaluation of coal mine safety production, Wang et al. [18] combined the particle swarm optimization (PSO) algorithm and the BP neural network and proposed the MPSO-BP network model, which they used for the early-warning analysis of coal mine safety production. Their results show that the new model can not only effectively reduce the possibility of a network falling into a local minimum, but also has the advantages of fast convergence and high precision. Wang et al. [19] used the nonlinear method to find the priority of coal mine production risk factors, analyzed the data by using the logarithmic fuzzy preference planning method, and compared it with the traditional EA method. Zhang et al. [20] proposed a method of predicting lava features before and after the tunnel construction through the selection of nine factors as the state of the underground lava evaluation index. Then, they used fuzzy AHP to determine the index weight according to the principle of fuzzy transformation and maximum membership degree principle in order to determine the underground lava state level, thereby proving its feasibility in terms of cloak mountain tunnel engineering applications. Sweta et al. [21] proposed a new Type 2 fuzzy logic system (T2FLS) for underground fire intensity prediction and risk assessment in coal mines. They verified the rationality of the model by statistical analysis and multiple linear regression analysis.

Although the above methods can perform the quantitative analysis of factors of uncertainty, these are rarely used in the field of TBM construction in a long coal mine slope. This is due to the huge risks involved in the TBM construction characterized by vagueness and uncertainty. Given that the existing models are unable to describe an effective mechanism describing the evolution of the uncertainty and risk simulation, a systematic and comprehensive study must be done to come up with a TBM construction risk control evaluation method for a coal mine slope.

In practical application, fuzzy sets only consider the membership information of things; they do not consider the non-membership information, which leads to an incomplete representation of things and restricts the reliability of the research results [22]. To overcome such a shortcoming, Chen et al. [23] proposed vague sets and introduced the concept of non-membership. The vague set is able to express the information of things in a more comprehensive way, resulting in the theory's rapid development in the field of decision-making [24-27]. Liu et al. [28] proposed the interval vague set, whereas Galindo et al. [29] combined the rough set with the vague set, thus proposing the rough vague set. Khaleed et al. [30] combined the soft set with the vague set and proposed the concept of the soft vague set. In view of the increasing complexity of practical engineering problems, the information of things is described more comprehensively by a trapezoidal vague number; thus, it is meaningful to study it in depth. To do so, it is necessary to introduce the concepts of trapezoidal Vague number distance, BM operator, weighted arithmetic average operator. It is also important to establish the method of maximum deviation model to determine the index weight, which will allow us to solve the problem of information synthesis risk monitoring in coal mine inclined wells.

To sum up, there are major shortcomings in the existing methods of coal mine construction risk assessment. First, the index system is not perfect. Second, the index is mostly a static attribute, and the subjective determination of weight is too large. Third, the selection of the index value is mostly a real number, and many parameter values in actual engineering are often interval values with ambiguity. Therefore, by perfecting the index system of a construction risk assessment and solving the problem of uncertain safety evaluation information of TBM construction in a coal mine inclined shaft, this study uses the trapezoidal Vague number to depict the evaluation information. Moreover, this work proposes a prediction model of TBM construction risk of a coal mine inclined shaft through the quantitative analysis of group benefit value, individual regret value and benefit ratio. The proposed model can deal with uncertain information more accurately.

The rest of this study is organized as follows. In the third part, the trapezoidal vague set and its properties are introduced, and the index system of TBM construction risk 
identification and evaluation is discussed. Furthermore, the VIKOR model of the construction risk prediction of a long inclined shaft is established based on the trapezoidal vague set. In Part 4, the model method is applied in a case study in order to evaluate the construction risks. Finally, Part 5 presents the conclusions of this work.

\section{Methodology}

\subsection{Preliminary concepts}

\subsubsection{The concept of vague sets}

The vague set has been previously defined in the literature [30]. Let $U=\left\{u_{1}, u_{2}, u_{3}, \ldots, u_{n}\right\}$ be our domain. The vague set $A$ on $U$ corresponds to two mappings, namely,

$$
t_{A}: U \rightarrow[0,1] f_{A}: U \rightarrow[0,1]
$$

where $0 \leq t_{A}\left(u_{i}\right)+f_{A}\left(u_{i}\right) \leq 1$ is required; $t_{A}\left(u_{i}\right)$ and $f_{A}\left(u_{i}\right)$ represent the membership and non-membership of evidence supporting and opposing $u \in A$, respectively; and $\pi_{A}\left(u_{i}\right)=1-t_{A}\left(u_{i}\right)-f_{A}\left(u_{i}\right)$ is called the hesitation degree. The larger $\pi_{A}\left(u_{i}\right)$ is, the more unknown information $A$ is. When $\pi_{A}\left(u_{i}\right)=0$, the vague set is reduced to the fuzzy set. Generally, the extent to which $u_{i}$ belongs to the vague set $A$ is denoted as the interval $\left[t_{A}\left(u_{i}\right), 1-f_{A}\left(u_{i}\right)\right]$.

\subsubsection{Trapezoidal vague number}

Let $A$ be the vague set on $U$, and $\tilde{\alpha}$ is a vague number in $A$, if

$t_{\tilde{\alpha}}(u)=\left\{\begin{array}{cl}\frac{u-a}{b-a} t_{\tilde{\alpha}}, & a \leq u \leq b \\ t_{\tilde{\alpha}}, & b \leq u \leq c \\ \frac{d-u}{d-c} t_{\tilde{\alpha}}, & c \leq u \leq d \\ 0, & \text { other }\end{array}\right.$

$f_{\tilde{\alpha}}(u)=\left\{\begin{array}{cr}\frac{b-u+\left(u-a_{1}\right) f_{\tilde{\alpha}}}{b-a_{1}}, & a_{1} \leq u \leq b \\ f_{\tilde{\alpha}}, & b \leq u \leq c \\ \frac{u-c+\left(d_{1}-u\right) f_{\tilde{\alpha}}}{d_{1}-c} t_{\tilde{\alpha}}, & c \leq u \leq d_{1} \\ 0, & \text { other }\end{array}\right.$

In the above, $0 \leq t_{\tilde{\alpha}} \leq 1,0 \leq f_{\tilde{\alpha}} \leq 1, t_{\tilde{\alpha}}+f_{\tilde{\alpha}} \leq 1$, and $a_{1} \leq a \leq b \leq c \leq d \leq d_{1}$, can be expressed as

$\tilde{\alpha}=\left\langle\left([a, b, c, d] ; t_{\tilde{\alpha}}\right),\left(\left[a_{1}, b, c, d_{1}\right] ; 1-f_{\tilde{\alpha}}\right)\right\rangle$

which is a trapezoidal vague number.

Normally, we take $[a, b, c, d]=\left[a_{1}, b, c, d_{1}\right]$, so we have $\tilde{\alpha}=\left([a, b, c, d] ; t_{\tilde{\alpha}}, 1-f_{\tilde{\alpha}}\right)$. The trapezoidal vague numbers examined in this study are all defined in the above cases, and the uniform hypothesis $a>0$ is also assumed for the convenience of the study.

In order to solve the practical engineering problems with trapezoidal vague numbers, the algorithm between these numbers should be given.

\subsubsection{The operation of the trapezoidal vague number} Let

$$
\begin{aligned}
& \tilde{\alpha}_{1}=\left(\left[a_{1}, b_{1}, c_{1}, d_{1}\right] ; t_{\tilde{\alpha}_{1}}, 1-f_{\tilde{a}_{1}}\right), \\
& \tilde{\alpha}_{2}=\left(\left[a_{2}, b_{2}, c_{2}, d_{2}\right] ; t_{\tilde{\alpha}_{2}}, 1-f_{\tilde{a}_{2}}\right),
\end{aligned}
$$

then

1) $\tilde{\alpha}_{1} \oplus \tilde{\alpha}_{2}=\left(\left[a_{1}+a_{2}, b_{1}+b_{2}, c_{1}+c_{2}, d_{1}+d_{2}\right]\right.$;

$$
\left.t_{\tilde{\alpha}_{1}}+t_{\tilde{\alpha}_{2}}-t_{\tilde{\alpha}_{1}} \cdot t_{\tilde{\alpha}_{2}},\left(1-f_{\tilde{\alpha}_{1}}\right)\left(1-f_{\tilde{\alpha}_{2}}\right)\right) \text {; }
$$

2) $\tilde{\alpha}_{1} \otimes \tilde{\alpha}_{2}=\left(\left[a_{1} a_{2}, b_{1} b_{2}, c_{1} c_{2}, d_{1} d_{2}\right]\right.$

$$
\left.t_{\tilde{\alpha}_{1}} t_{\tilde{\alpha}_{2}}, 1-f_{\tilde{\alpha}_{1}} \cdot f_{\tilde{\alpha}_{2}}\right) \text {; }
$$

3) $\lambda \tilde{\alpha}_{1}=\left(\left[\lambda a_{1}, \lambda b_{1}, \lambda c_{1}, \lambda d_{1}\right] ; 1-\left(1-t_{\tilde{\alpha}}\right)^{\lambda},\left(1-f_{\tilde{\alpha}}\right)^{\lambda}\right)$, here $\lambda \geq 0$

4) $\left(\tilde{\alpha}_{1}\right)^{\lambda}=\left(\left[a_{1}^{\lambda}, b_{1}^{\lambda}, c_{1}^{\lambda}, d_{1}^{\lambda}\right] ;\left(t_{\tilde{\alpha}_{1}}\right)^{\lambda}, 1-f_{\tilde{\alpha}_{1}}^{\lambda}\right)$, here $\lambda \geq 0$.

\subsubsection{The distance of the trapezoidal vague number} The distance between $\tilde{\alpha}_{1}$ and $\tilde{\alpha}_{2}$ is specified below.

$$
\begin{aligned}
d\left(\tilde{\alpha}_{1}, \tilde{\alpha}_{2}\right)= & \frac{1}{8}\left(\left|\left(t_{\tilde{\alpha}_{1}}+1-f_{\tilde{\alpha}_{1}}\right) a_{1}-\left(t_{\tilde{\alpha}_{2}}+1-f_{\tilde{\alpha}_{2}}\right) a_{2}\right|\right. \\
& +\left|\left(t_{\tilde{\alpha}_{1}}+1-f_{\tilde{\alpha}_{1}}\right) b_{1}-\left(t_{\tilde{\alpha}_{2}}+1-f_{\tilde{\alpha}_{2}}\right) b_{2}\right|+ \\
& +\left|\left(t_{\tilde{\alpha}_{1}}+1-f_{\tilde{\alpha}_{1}}\right) c_{1}-\left(t_{\tilde{\alpha}_{2}}+1-f_{\tilde{\alpha}_{2}}\right) c_{2}\right|+ \\
& \left.\left|\left(t_{\tilde{\alpha}_{1}}+1-f_{\tilde{\alpha}_{1}}\right) d_{1}-\left(t_{\tilde{\alpha}_{2}}+1-f_{\tilde{\alpha}_{2}}\right) d_{2}\right|\right)
\end{aligned}
$$

3.2 Integration operator of the trapezoidal vague number 3.2.1 Trapezoidal vague $B M$ operator

Let $p, q>0$, the non-negative set of real numbers $\left\{x_{1}, x_{2}, \cdots x_{n}\right\}$, if

$B^{p, q}\left(x_{1}, x_{2}, \cdots x_{n}\right)=\left(\frac{1}{n(n-1)} \sum_{\substack{i, j=1 \\ i \neq j}}^{n} x_{i}^{p} x_{j}^{q}\right)^{\frac{1}{p+q}}$

where $B^{p, q}$ is the BM operator.

In order to deal with the trapezoidal vague number decision problem, the following is combined with the trapezoidal vague number $\mathrm{BM}$ operator.

Let $A$ be the $\tilde{\alpha}_{i}=\left(\left[a_{i}, b_{i}, c_{i}, d_{i}\right] ; t_{\tilde{\alpha}_{i}}, 1-f_{\tilde{\alpha}_{i}}\right)$, $i=(1,2, \cdots n)$ group of trapezoidal vague numbers, and $p, q>0$, then the trapezoidal vague $\mathrm{BM}$ operator is expressed as 
$\operatorname{TFNVGBM} M^{p, q}\left(\tilde{\alpha}_{1}, \tilde{\alpha}_{2}, \cdots \tilde{\alpha}_{n}\right)=\left(\frac{1}{n(n-1)}\left(\bigoplus_{\substack{i, j=1 \\ i \neq j}}^{n}\left(\tilde{\alpha}_{i}^{p} \otimes \tilde{\alpha}_{j}^{q}\right)\right)\right)^{\frac{1}{p+q}}$

For the convenience of research, $\tilde{\alpha}_{i}$ is abbreviated as $\tilde{\alpha}_{i}=\left(\left[a_{i}, b_{i}, c_{i}, d_{i}\right] ; t_{i}, 1-f_{i}\right)$ below.

Set $\quad \tilde{\alpha}_{i}=\left(\left[a_{i}, b_{i}, c_{i}, d_{i}\right] ; t_{i}, 1-f_{i}\right)$ as a group of trapezoidal vague numbers and $p, q>0$, then it remains a trapezoidal vague number after the integrated operation of Equation (6), that is,

$\operatorname{TFNVGBM} M^{p, q}\left(\tilde{\alpha}_{1}, \tilde{\alpha}_{2}, \cdots \tilde{\alpha}_{n}\right)=\left([a, b, c, d] ; t_{\tilde{\alpha}}, 1-f_{\tilde{\alpha}}\right)$.

In the formula,

$a=\left(\frac{1}{n(n+1)} \sum_{\substack{i, j=1 \\ i \neq j}}^{n} a_{i}^{p} \cdot a_{j}^{q}\right)^{\frac{1}{p+q}}$,

$b=\left(\frac{1}{n(n+1)} \sum_{\substack{i, j=1 \\ i \neq j}}^{n} b_{i}^{p} \cdot b_{j}^{q}\right)^{\frac{1}{p+q}}$

$c=\left(\frac{1}{n(n+1)} \sum_{\substack{i, j=1 \\ i \neq j}}^{n} c_{i}^{p} \cdot c_{j}^{q}\right)^{\frac{1}{p+q}}$,

$d=\left(\frac{1}{n(n+1)} \sum_{\substack{i, j=1 \\ i \neq j}}^{n} d_{i}^{p} \cdot d_{j}^{q}\right)^{\frac{1}{p+q}}$,

$t_{\tilde{\alpha}}=\left(1-\prod_{\substack{i, j=1 \\ i \neq j}}^{n}\left(1-t_{i}^{p} \cdot t_{j}^{q}\right)^{\frac{1}{n(n-1)}}\right)^{\frac{1}{p+q}}$,

$1-f_{\tilde{\alpha}}=\left(1-\prod_{\substack{i, j=1 \\ i \neq j}}^{n}\left(1-t_{i}^{p} \cdot t_{j}^{q}\right)^{\frac{1}{n(n-1)}}\right)^{\frac{1}{p+q}}$

The above TFNVGBM operator is a new integration method, and the problem of inter-attribute correlation is comprehensively considered. However, in practical engineering applications, the weight of various attributes is not the same. Therefore, a trapezoidal vague number weighted arithmetic mean operator is proposed in this study.

3.2.2 Trapezoidal vague number weighted arithmetic mean operator

Let $\quad \tilde{\alpha}_{1}=\left(\left[a_{1}, b_{1}, c_{1}, d_{1}\right] ; t_{1}, 1-f_{1}\right) \quad$ and

$\tilde{\alpha}_{2}=\left(\left[a_{2}, b_{2}, c_{2}, d_{2}\right] ; t_{2}, 1-f_{2}\right)$ be two groups of trapezoidal vague numbers, then

1) $\tilde{\alpha}_{1}+\tilde{\alpha}_{2}=\left(\left[a_{1}+a_{2}, b_{1}+b_{2}, c_{1}+c_{2}, d_{1}+d_{2}\right]\right.$;

$$
\left.t_{1}+t_{2}-t_{2} t_{2}, 1-f_{1} f_{2}\right)
$$

2) $\lambda \tilde{\alpha}_{1}=\left(\left[\lambda a_{1}, \lambda b_{1}, \lambda c_{1}, \lambda d_{1}\right] ; 1-\left(1-t_{1}\right)^{\lambda}, 1-f_{1}^{\lambda}\right), \lambda>0$.
3) $\tilde{\alpha}_{1} \tilde{\alpha}_{2}=\left(\left[a_{1} a_{2}, b_{1} b_{2}, c_{1} c_{2}, d_{1} d_{2}\right] ; t_{1} t_{2}, 1-f_{1}-f_{2}+f_{1} f_{2}\right)$;

4) $\left(\tilde{\alpha}_{1}\right)^{\lambda}=\left(\left[a_{1}^{\lambda}, b_{1}^{\lambda}, c_{1}^{\lambda}, d_{1}^{\lambda}\right] ; t_{1}^{\lambda},\left(1-f_{1}\right)^{\lambda}\right), \lambda \geq 0$.

If $\tilde{\alpha}_{i}=\left(\left[a_{i}, b_{i}, c_{i}, d_{i}\right] ; t_{i}, 1-f_{i}\right),(i=1,2, \cdots, n)$ is a set of trapezoidal Vague Numbers, $w=\left(w_{1}, w_{2}, \cdots, w_{n}\right)$ is the weight set $0 \leq w_{i} \leq 1$ corresponding to $\tilde{\alpha}_{i}$, and $\sum_{i=1}^{n} w_{i}=1$, then

$$
f_{w}\left(\tilde{\alpha}_{1}, \tilde{\alpha}_{2}, \cdots \tilde{\alpha}_{n}\right)=\sum_{i=1}^{n} w_{i} \tilde{\alpha}_{i}
$$

is the weighted arithmetic mean operator $\tilde{\alpha}_{i},(i=1,2, \cdots, n)$.

Assume $\tilde{\alpha}_{i}=\left(\left[a_{i}, b_{i}, c_{i}, d_{i}\right] ; t_{i}, 1-f_{i}\right),(i=1,2, \cdots, n)$ is a set of trapezoidal vague numbers, then the results of the integration of formula (8) are still trapezoidal vague numbers; hence,

$$
\begin{aligned}
& f_{w}\left(\tilde{\alpha}_{1}, \tilde{\alpha}_{2}, \cdots \tilde{\alpha}_{n}\right)=\left(\left[\sum_{i=1}^{n} w_{i} a_{i}, \sum_{i=1}^{n} w_{i} b_{i}, \sum_{i=1}^{n} w_{i} c_{i}, \sum_{i=1}^{n} w_{i} d_{i}\right] ;\right. \\
&\left.1-\prod_{i=1}^{n}\left(1-t_{i}\right)^{w_{i}}, 1-\prod_{i=1}^{n} f_{i}^{w_{i}}\right)
\end{aligned}
$$

\subsection{Identification of TBM construction risk of a long} inclined shaft in a coal mine.

Through the analysis of the TBM construction risk of a long inclined shaft in a coal mine, 27 common factors in the construction were identified, and a two-level risk assessment index system was established in this study, as shown in Table 1. The first-level risk assessment index takes into account the five main factors in the TBM construction process of a long inclined shaft in a coal mine, namely, the natural, geological, technical, equipment, and internal factors. The second-level risk assessment index is based on the existing research, with the addition of rock burst factors, collapse factors, TBM card machines, and toxic gas poisoning factors. At the same time, the TBM construction risk of a long inclined shaft in a coal mine is divided into four levels, as shown in Table 2.

3.4 Risk prediction VIKOR model for the TBM construction of a long inclined shaft in a coal mine In the construction risk analysis, $Y=\left\{Y_{1}, Y_{2}, Y_{3}, Y_{4}\right\}$ is the risk level set, and 4 is the number of levels. We define the risk assessment factor set as $A=\left\{B_{1}, B_{2}, B_{3}, B_{4}, B_{5}\right\}$, which has a total of 5 indicators. A total of 27 secondary indicators can be found in the first level, which include the following: $B_{1}=\left\{C_{11}, C_{12}, C_{13}, C_{14}, C_{15}\right\} \quad, \quad B_{2}=\left\{C_{21}, C_{22}, C_{23}, C_{24}, C_{25}\right\} \quad$, $B_{3}=\left\{C_{31}, C_{32}, C_{33}, C_{34}, C_{35}, C_{36}\right\}, B_{4}=\left\{C_{41}, C_{42}, C_{43}, C_{44}, C_{45}, C_{46}\right\}$, and $B_{5}=\left\{C_{51}, C_{52}, C_{53}, C_{54,}, C_{55}\right\} \quad$. In addition, $\tilde{F}\left(B_{g}\right)=\left(\tilde{\alpha}_{i j}^{g}\right)_{4 n},(g=1,2, \cdots 5)$ is the trapezoidal vague number evaluation matrix in which $\tilde{\alpha}_{i j}^{g},(i=1,2,3,4 ; j=1,2, \cdots n)$ is the trapezoidal vague number corresponding to the first level indicator $g$ and the second level indicator $j$ in risk level $Y_{i}$. 
Table 1. The two-level risk assessment index system of a long inclined shaft in a coal mine

\begin{tabular}{|c|c|c|}
\hline The target layer & The first-level index & The second-level index \\
\hline \multirow{5}{*}{$\begin{array}{l}\text { Risk assessment system of } \\
\text { a long inclined shaft in a } \\
\text { coal mine } A\end{array}$} & Natural factors $B_{1}$ & $\begin{array}{c}\text { Landslide } C_{11} \\
\text { Flood } C_{12} \\
\text { Rainy season } C_{13} \\
\text { Impact pressure } C_{14} \\
\text { The harmful gas } C_{15} \\
\end{array}$ \\
\hline & Geological factors $B_{2}$ & $\begin{array}{c}\text { Landslide risk } C_{21} \\
\text { Water risk } C_{22} \\
\text { Wall rock plastic deformation } C_{23} \\
\text { Rockburst risk } C_{24} \\
\text { Special area } C_{25} \\
\end{array}$ \\
\hline & Technical factors $B_{3}$ & $\begin{array}{c}\text { Inclined shaft axis positioning deviation is too large } C_{31} \\
\text { Improper control of grouting parameters } C_{32} \\
\text { Drainage problem } C_{33} \\
\text { Ventilation problem } C_{34} \\
\text { Application of new technology } C_{35} \\
\text { Gas explosion } C_{36}\end{array}$ \\
\hline & Equipment factors $B_{4}$ & $\begin{array}{c}\text { Shield machine is damaged } C_{41} \\
\text { TBM card machine } C_{42} \\
\text { TBM is improperly selected for } C_{43} \\
\text { Improper installation and maintenance of motor equipment } C_{44} \\
\text { Device debugging error } C_{45} \\
\text { Fire } C_{46}\end{array}$ \\
\hline & Internal factors $B_{5}$ & $\begin{array}{c}\text { Managerial personnel are not qualified } C_{51} \\
\text { Production management system is not perfect } C_{52} \\
\text { Material shortage } C_{53} \\
\text { Construction design unreasonable } C_{54} \\
\text { Material price changes } C_{55}\end{array}$ \\
\hline
\end{tabular}

\begin{tabular}{c|c} 
Table.2. Risk rating scale & Impact on the project \\
\hline Risk level & There is a slight risk; the risk is low \\
\hline First-order risk $Y_{1}$ & High risk; has a certain influence to the project \\
The secondary risk $Y_{2}$ & The level of risk is high and can cause serious damage to the project \\
Three levels of risk $Y_{3}$ & The highest level of risk can be devastating to the project \\
Four levels of risk $Y_{4}$ &
\end{tabular}

\subsubsection{Determination of the index weight}

In order to solve the objective weight determination method under the condition that the weight information is completely unknown, we use the idea of maximizing the deviation to determine the weight. For a multi-attribute decision problem, the greater the deviation value of an index for different schemes, the greater the weight of that index. However, if an index is the same as the assignment of all schemes, the weight of the index can be considered as 0 .

Let us suppose that the weight corresponding to the second-level indicator $C_{g j}$ under the first-level indicator $B_{g}$ is $w_{g j}$, and the deviation of risk level $Y_{i}$ for all other levels is $D_{i j}=\sum^{m} d\left(\tilde{\alpha}_{k j}^{g}, \tilde{\alpha}_{i j}^{g}\right) w_{g j}$, where $i=1,2, \cdots, m ; \quad j=1,2, \cdots, n$; $g=1,2, \cdots, 5$. For the secondary index $C_{g j}$, the deviation value of all risk levels to other levels is $D_{j}\left(w_{g j}\right)=\sum_{i=1}^{m} \sum_{k=1}^{m} d\left(\tilde{\alpha}_{k j}^{g}, \tilde{\alpha}_{i j}^{g}\right) w_{g j}$, and the total deviation value of all secondary indicators at all risk levels is the $\max D\left(w_{g j}\right)=\sum_{j=1}^{n} \sum_{i=1}^{m} \sum_{k=1}^{m} d\left(\tilde{\alpha}_{k j}^{g}, \tilde{\alpha}_{i j}^{g}\right) w_{g j}$. On the basis of such an analysis, the following nonlinear model can be constructed to obtain the weight $w_{g j}$, which is defined as follows:

$\max D\left(w_{g j}\right)=\sum_{j=1}^{n} \sum_{i=1}^{m} \sum_{k=1}^{m} d\left(\tilde{\alpha}_{k j}^{g}, \tilde{\alpha}_{i j}^{g}\right) w_{g j}$ 
where $\sum_{j}^{n} w_{g j}^{2}=1$.

Next, we construct a Lagrangian function $L\left(\lambda, w_{g j}\right)=\sum_{j=1}^{n} \sum_{i=1}^{m} \sum_{k=1}^{m} d\left(\tilde{\alpha}_{k j}^{g}, \tilde{\alpha}_{i j}^{g}\right) w_{g j}+\frac{\lambda}{2}\left(\sum_{j=1}^{n} w_{g j}^{2}-1\right)$ and take the partial derivative of $L\left(\lambda, w_{g j}\right)$ with respect to $\lambda, w_{g j}$, that is,

$$
\left\{\begin{array}{l}
\frac{\partial L\left(\lambda, w_{g j}\right)}{\partial w_{g j}}=\sum_{i=1}^{m} \sum_{k=1}^{m} d\left(\tilde{\alpha}_{k j}^{g}, \tilde{\alpha}_{i j}^{g}\right)+\lambda w_{g j}=0 \\
\frac{\partial L\left(\lambda, w_{g j}\right)}{\partial \lambda}=\frac{1}{2}\left(\sum_{j=1}^{n} w_{g j}^{2}-1\right)=0
\end{array}\right.
$$

The weight vector $w_{g}=\left(w_{g 1}, w_{g 2}, \cdots, w_{g n}\right)$ corresponding to the secondary index $C_{g j}(j=1,2, \cdots, n)$ is obtained, and

$$
w_{g j}=\frac{\sum_{i=1}^{m} \sum_{k=1}^{m} d\left(\tilde{\alpha}_{k j}^{g}, \tilde{\alpha}_{i j}^{g}\right)}{\sqrt{\sum_{j=1}^{n}\left(\sum_{i=1}^{m} \sum_{k=1}^{m} d\left(\tilde{\alpha}_{k j}^{g}, \tilde{\alpha}_{i j}^{g}\right)\right)^{2}}}
$$

is normalized to

$$
w_{g j}^{*}=\frac{w_{g j}}{\sum_{j=1}^{n} w_{g j}}
$$

Similarly, the weight vector $w^{*}=\left(w_{1}^{*}, w_{2}^{*}, \cdots, w_{5}^{*}\right)$ corresponding to the first-order index $B_{g}(g=1,2, \cdots, 5)$ can be obtained.

\subsubsection{VIKOR decision method based on the trapezoidal vague number}

In multi-attribute decision theory, VIKOR and TOPSIS are two common decision methods, both of which aim to find the optimal solution by finding the positive and negative ideal solution in the solution set. In practical engineering problems, although the former is not as widely used as the latter, the optimal solution obtained by the VIKOR method is closer to the ideal solution.

In the actual construction analysis, there are $m$ risk levels to be evaluated and $n$ influencing factors (indicators); thus, the trapezoidal vague number corresponding to the $j$ attribute value in the $i$ risk level is $\tilde{\alpha}_{i j}$, which is defined as $\tilde{\alpha}_{i j}=\left(\left[a_{i j}, b_{i j}, c_{i j}, d_{i j}\right] ; t_{i j}, 1-f_{i j}\right)$. The respective positive and negative ideal solutions $\tilde{\alpha}^{+}$and $\tilde{\alpha}^{-}$are

$\tilde{\alpha}^{+}=\left\{r_{1}, r_{2}, \cdots, r_{n}\right\}, \quad \tilde{\alpha}^{-}=\left\{h_{1}, h_{2}, \cdots, h_{n}\right\}$

In the formula,

$r_{j}=\left\{\begin{array}{l}\max _{i} \tilde{\alpha}_{i j}, j \in I_{1} \\ \min _{i} \tilde{\alpha}_{i j}, j \in I_{2}\end{array}, h_{j}=\left\{\begin{array}{l}\min _{i} \tilde{\alpha}_{i j}, j \in I_{1} \\ \max _{i} \tilde{\alpha}_{i j}, j \in I_{2}\end{array}\right.\right.$.
Here $I_{1}$ and $I_{2}$ are the benefit and cost attributes, respectively. In addition,

$$
\begin{aligned}
& \max _{i} \tilde{\alpha}_{i j}=\left(\left[\max _{i} a_{i j}, \max _{i} b_{i j}, \max _{i} c_{i j}, \max _{i} d_{i j}\right] ; \max _{i} t_{i j}, \max _{i}\left(1-f_{i j}\right) ;\right. \\
& \min _{i} \tilde{\alpha}_{i j}=\left(\left[\min _{i} a_{i j}, \min _{i} b_{i j}, \min _{i} c_{i j}, \min _{i} d_{i j}\right] ; \min _{i} t_{i j}, \min _{i}\left(1-f_{i j}\right) .\right.
\end{aligned}
$$

First, the group benefit value $S_{i}$ and individual regret value $R_{i}(i=1,2, L, m)$ of each risk level are calculated respectively as

$$
\begin{aligned}
& S_{i}=\sum_{j=1}^{n} w_{j}^{*} d\left(r_{j}, \tilde{\alpha}_{i j}\right) / d\left(r_{j}, h_{j}\right) \\
& R_{i}=\max _{j}\left(w_{j}^{*} d\left(r_{j}, \tilde{\alpha}_{i j}\right) / d\left(r_{j}, h_{j}\right)\right)
\end{aligned}
$$

where $w_{i}^{*}$ is the weight of the $j$ index. When the value $S_{i}$ is smaller, the corresponding population effect is larger. The smaller the value of $R_{i}$, the smaller the individual regret.

Then, we used $S_{i}$ and $R_{i}$ to determine the benefit ratio $Q_{i}$ for each risk level

$$
Q_{i}=\delta\left(S_{i}-S^{-}\right) /\left(S^{+}-S^{-}\right)+(1-\delta)\left(R_{i}-R^{-}\right) /\left(R^{+}-R^{-}\right)
$$

where $\delta$ is the decision mechanism coefficient, $S^{-}=\min _{i} S_{i}, S^{+}=\max _{i} S_{i}, R^{-}=\min _{i} R_{i}$, and $R^{+}=\max _{i} R_{i}$.

If $\delta>0.5$, this indicates that the group benefit plays a leading role in the decision-making process. If $\delta<0.5$, it indicates that individual regret values play an important role in the decision-making process. Finally, if $\delta=0.5$, this shows that decisions are made through balanced tradeoffs. The smaller the value of $Q_{i}(i=1,2, L, m)$ is, the better the scheme would be.

Notably, sorting by $Q_{i}$ must be able to meet two conditions. First, the distance between the two smallest adjacent $Q_{i}$ values should meet the minimum advantage value, that is, the distance must be greater than or equal to $1 /(m-1)$. Second, a high degree of decision reliability must be ensured, that is, the ranking of $Q_{i}, S_{i}$, and $R_{i}$ should be consistent. However, the minimum advantage value cannot be guaranteed to be established in the actual decision; thus, the level corresponding to $Q_{i}$ the minimum value can only be selected as the optimal risk level.

The decision steps are as follows:

1) Formula (12) is used to calculate the weight $w_{g i}^{*}$ of each secondary index.

2) The trapezoidal vague number of the first-order index is obtained by integrating the second-order index with Formula (9).

3) Formula (13) is used to determine the positive and negative ideal solutions $\tilde{\alpha}^{+}$and $\tilde{\alpha}^{-}$, respectively, in the risk rating concentration.

4) Formula (12) is used to calculate the weight $w_{g}^{*}$ of each first-level index.

5) The group benefit value $S_{i}$, individual regret value $R_{i}$, and benefit ratio $Q_{i}$ for each risk level are all calculated by using Equations (14), (15), and (16), respectively. 
6) The smaller the value of $Q_{i}$, the better the risk level.

\section{Result analysis and discussion}

\subsection{Case study}

The Taigmiao mining area of the Shenhua Group is located in the south-central Ordos City, which has typical plateau accumulation-type hilly landform characteristics without surface water system development. It belongs to weak earthquake area with intensity of VI degrees. The mining area comprises mainly of siltstone and sandy mudstone. For rock, the porosity ranges from $0.72 \%-23.74 \%$, the moisture content ranges from $0.09 \%-4.03 \%$, the Prussian coefficients are between 1.1 and 10.4 , and the tensile strength ranges from 1.04-3.66 MPa. The elevation of the test well head is $1320 \mathrm{~m}$ and that of the bottom is $660 \mathrm{~m}$. The length of the TBM construction section is $6109 \mathrm{~m}$, and its radius is 3.65 $\mathrm{m}$. From top to bottom, the construction section passes through the strata of Lower Cretaceous Zhidan Group, the Middle Jurassic Anding Group, and the Middle Jurassic Yanan Group.

The construction risk assessment of the long inclined shaft (No. 2 test well) in the Taigemiao mining area of the Shenhua Group was carried out in accordance with the TBM construction risk assessment index system (Table 2). After consulting the construction materials and asking experts' opinions, the evaluation values of the four risk levels $Y_{i}(i=1,2,3,4)$ under different indicators were obtained and subsequently expressed as trapezoidal vague number. The results are shown in Tables 3-7.

\subsection{Determination of the index weight and information} aggregation

According to Equation (12), the weight of each secondary index is determined.

$$
\begin{aligned}
& w_{B_{1}}^{*}=(0.1477,0.212,0.0413,0.3906,0.2084), \\
& w_{B_{2}}^{*}=(0.2893,0.1335,0.1573,0.2473,0.1726), \\
& w_{B_{3}}^{*}=(0.1206,0.1381,0.1202,0.1589,0.2366), \\
& w_{B_{4}}^{*}=(0.1987,0.2108,0.1508,0.2462,0.1006,0.0929), \\
& w_{B_{5}}^{*}=(0.1115,0.2054,0.2943,0.327,0.0618) .
\end{aligned}
$$

According to Formula (12), the weight of each firstlevel index is determined:

$$
w^{*}=(0.1412,0.2053,0.1582,0.2279,0.2674) .
$$

Formula (9) was used to aggregate the secondary indicator information to obtain the comprehensive trapezoidal vague number information, as shown in Table 8.

Table 3. The trapezoidal vague evaluation value of risk levels $Y_{i}$ in each natural factor $B_{1}$

\begin{tabular}{c|c|c|c|c|c}
\hline & $C_{11}$ & $C_{12}$ & $C_{13}$ & $C_{14}$ & $C_{15}$ \\
\hline$Y_{1}$ & $([1,2,5,7] ; 0.4,0.5)$ & $([3,5,6,7] ; 0.4,0.6)$ & $([2,3,4,5] ; 0.3,0.4)$ & $([1,2,5,6] ; 0.4,0.5)$ & $([2,3,4,6] ; 0.5,0.6)$ \\
$Y_{2}$ & $([2,3,5,7] ; 0.2,0.4)$ & $([1,2,3,4] ; 0.3,0.4)$ & $([2,3,4,5] ; 0.2,0.4)$ & $([3,4,6,7] ; 0.7,0.8)$ & $([2,3,4,5] ; 0.6,0.7)$ \\
$Y_{3}$ & $([4,5,6,7] ; 0.3,0.5)$ & $([1,3,4,6] ; 0.4,0.5)$ & $([1,2,3,5] ; 0.4,0.5)$ & $([4,5,6,7] ; 0.4,0.5)$ & $([1,2,3,4] ; 0.6,0.7)$ \\
$Y_{4}$ & $([1,2,5,7] ; 0.2,0.4)$ & $([2,3,5,7] ; 0.3,0.6)$ & $([2,3,4,5] ; 0.3,0.4)$ & $([1,2,3,4] ; 0.2,0.3)$ & $([3,4,5,6] ; 0.7,0.8)$ \\
\hline
\end{tabular}

Table 4. The trapezoidal vague evaluation value of risk levels $Y_{i}$ in each geological factor $B_{2}$

\begin{tabular}{|c|c|c|c|c|c|c|}
\hline & $C_{41}$ & $C_{42}$ & $C_{43}$ & $C_{44}$ & $C_{45}$ & $C_{46}$ \\
\hline $\begin{array}{l}Y_{1} \\
Y_{2}\end{array}$ & $\begin{array}{c}{[2,3,5,6] ;} \\
0.2,0.5) \\
([1,4,6,8] ; \\
0.3,0.6) \\
([2,3,5,7] ; \\
0.1,0.3)\end{array}$ & $\begin{array}{c}([3,4,5,7] ; \\
0.5,0.8) \\
([2,3,6,8] ; \\
0.2,0.5) \\
([1,2,4,8] ; \\
0.1,0.3)\end{array}$ & $\begin{array}{c}{[1,4,5,6] ;} \\
0.2,0.4) \\
([2,4,5,7] ; \\
0.3,0.9) \\
([2,3,5,6] ; \\
0.2,0.5)\end{array}$ & $\begin{array}{c}{[1,2,3,5]} \\
0.2,0.7) \\
([2,3,4,8] \\
0.3,0.6) \\
([3,4,5,6] \\
0.8,0.9)\end{array}$ & $\begin{array}{c}{[1,2,3,4] ;} \\
0.5,0.7) \\
([4,5,6,7] ; \\
0.4,0.5) \\
([2,3,4,5] ; \\
0.4,0.6)\end{array}$ & $\begin{array}{c}{[2,3,5,7] ;} \\
0.2,0.3) \\
([1,3,4,6] ; \\
0.3,0.6) \\
([1,4,5,7] ; \\
0.4,0.5)\end{array}$ \\
\hline
\end{tabular}

\begin{tabular}{c|c|c|c|c|c}
\hline & $C_{21}$ & $C_{22}$ & $C_{23}$ & $C_{24}$ & $C_{25}$ \\
\hline$Y_{1}$ & $([1,3,4,6] ; 0.3,0.9)$ & $([2,3,4,8] ; 0.4,0.6)$ & $([2,4,5,6] ; 0.3,0.5)$ & $([1,2,5,6] ; 0.4,0.6)$ & $([1,2,3,5] ; 0.1,0.3)$ \\
$Y_{2}$ & $([2,3,5,7] ; 0.1,0.4)$ & $([1,3,4,7] ; 0.2,0.4)$ & $([2,3,4,5] ; 0.3,0.4)$ & $([2,3,5,6] ; 0.7,0.8)$ & $([2,3,4,6] ; 0.5,0.6)$ \\
$Y_{3}$ & $([1,2,3,8] ; 0.2,0.5)$ & $([2,4,6,7] ; 0.3,0.5)$ & $([1,3,4,6] ; 0.6,0.8)$ & $([3,4,5,6] ; 0.8,0.9)$ & $([1,2,3,4] ; 0.4,0.5)$ \\
$Y_{4}$ & $([1,5,6,7] ; 0.6,0.8)$ & $([1,2,4,6] ; 0.4,0.6)$ & $([1,3,4,5] ; 0.3,0.5)$ & $([3,5,6,7] ; 0.4,0.6)$ & $([2,3,4,5] ; 0.2,0.4)$ \\
\hline
\end{tabular}

Table 5. The trapezoidal vague evaluation value of risk levels $Y_{i}$ in each technical factor $B_{3}$

\begin{tabular}{c|c|c|c|c|c|c}
\hline & $C_{31}$ & $C_{32}$ & $C_{33}$ & $C_{34}$ & $C_{35}$ & $C_{36}$ \\
\hline$Y_{1}$ & $([1,2,3,5] ; 0.4,0.8)$ & $([2,3,4,5] ; 0.6,0.8)$ & $([3,4,5,6] ; 0.4,0.7)$ & $([2,3,4,7] ; 0.3,0.8)$ & $([1,2,3,4] ; 0.5,0.6)$ & $([4,5,6,7] ; 0.4,0.6)$ \\
$Y_{2}$ & $([2,4,5,6] ; 0.4,0.7)$ & $([1,2,3,7] ; 0.3,0.5)$ & $([1,2,3,4] ; 0.2,0.3)$ & $([2,3,4,6] ; 0.4,0.7)$ & $([2,3,5,6] ; 0.2,0.3)$ & $([3,4,6,7] ; 0.8,0.9)$ \\
$Y_{3}$ & $([3,4,5,8] ; 0.3,0.7)$ & $([1,2,4,5] ; 0.4,0.9)$ & $([2,3,4,5] ; 0.6,0.8)$ & $([3,5,6,7] ; 0.4,0.7)$ & $([3,4,5,6] ; 0.5,0.6)$ & $([1,2,4,5] ; 0.6,0.8)$ \\
$Y_{4}$ & $([1,2,4,7] ; 0.4,0.8)$ & $([2,3,4,5] ; 0.3,0.7)$ & $([1,3,4,5] ; 0.4,0.5)$ & $([2,3,4,5] ; 0.4,0.6)$ & $([1,2,4,5] ; 0.4,0.7)$ & $([2,4,5,6] ; 0.5,0.6)$ \\
\hline
\end{tabular}

Table 6. The trapezoidal vague evaluation value of risk levels $Y_{i}$ in each equipment factor

$B_{4}$ 


\begin{tabular}{c|c|c|c|c|c|c} 
& \\
$Y_{4}$ & $([4,5,6,7] ;$ & $([2,4,5,7] ;$ & $([1,2,3,7] ;$ & $([1,3,4,8] ;$ & $([3,4,5,6] ;$ & $([1,3,4,5] ;$ \\
\hline
\end{tabular}

Table 7. The trapezoidal vague evaluation value of risk levels $Y_{i}$ in each internal factor $B_{5}$

\begin{tabular}{c|c|c|c|c|c}
\hline & $C_{51}$ & $C_{52}$ & $C_{53}$ & $C_{54}$ & $C_{55}$ \\
\hline$Y_{1}$ & $([3,4,5,6] ; 0.3,0.5)$ & $([1,2,4,6] ; 0.2,0.4)$ & $([1,2,5,7] ; 0.1,0.4)$ & $([1,2,5,6] ; 0.4,0.5)$ & $([2,3,5,6] ; 0.2,0.4)$ \\
$Y_{2}$ & $([2,3,4,5] ; 0.5,0.6)$ & $([4,5,6,7] ; 0.4,0.5)$ & $([2,5,6,8] ; 0.1,0.3)$ & $([3,4,5,6] ; 0.7,0.9)$ & $([2,3,4,5] ; 0.3,0.5)$ \\
$Y_{3}$ & $([1,3,5,7] ; 0.2,0.4)$ & $([1,3,5,7] ; 0.4,0.7)$ & $([3,4,5,6] ; 0.6,0.9)$ & $([2,3,4,5] ; 0.2,0.4)$ & $([1,2,3,5] ; 0.5,0.6)$ \\
$Y_{4}$ & $([1,2,3,4] ; 0.4,0.5)$ & $([2,3,5,6] ; 0.2,0.5)$ & $([1,3,5,6] ; 0.3,0.7)$ & $([3,4,6,7] ; 0.5,0.7)$ & $([1,2,3,4] ; 0.6,0.7)$ \\
\hline
\end{tabular}

Table 8. Comprehensive trapezoidal vague value evaluation information of the coal mine

\begin{tabular}{c|c|c|c|c|c}
\hline & $B_{1}$ & $B_{2}$ & $B_{3}$ & $B_{4}$ & $B_{5}$ \\
\hline$Y_{1}$ & $([3.256,5.61,9.652,12.289$ & $([2.769,5.8728,9.08,13.0$ & $([5.69,8.05,10.41,13.61]$ & $([3.97,6.98,9.98,13.57] ;$ & $([2.56,4.554,9.554,12.54] ;$ \\
& ]$; 0.652,0.703)$ & $7] ; 0.54,0.687)$ & $; 0.74,0.57)$ & $0.57,0.72)$ & $0.44,0.804)$ \\
$Y_{2}$ & $([4.23,6.181,9.172,11.404$ & $([4.004,6.436,9.733,13.4$ & $([4.41,7.05,10.34,13.95]$ & $([4.42,8.23,11.9,17.53] ;$ & $([5.45,8.62,10.61,13.19] ; 0$ \\
& ]$; 0.78,0.642)$ & $4] ; 0.68,0.76)$ & $; 0.77,0.79)$ & $0.54,0.69)$ & $.714,0.715)$ \\
$Y_{3}$ & $([5.0839,7.4416,9.3865,11$ & $([3.49,6.26,8.69,13.66] ;$ & $([4.77,7.41,10.64,13.57]$ & $([4.5,7.254,10.87,15.33]$ & $([3.816,6.44,9.065,11.81] ;$ \\
& $.82] ; 0.671,0.7)$ & $0.79,0.63)$ & $; 0.811,0.48)$ & $; 0.734,0.819)$ & $0.641,0.657)$ \\
$Y_{4}$ & $([3.248,5.193,8.12,10.7] ; 0$ & $([3.57,8.45,10.88,13.3] ;$ & $([3.521,6.965,9.98,12.9]$ & $([4.66,8.251,10.56,16.1$ & $([3.70,6.28,9.92,11.91] ; 0$. \\
& $.592,0.788)$ & $0.69,0.67)$ & $; 0.714,0.67)$ & $3] ; 0.643,0.82)$ & $625.602)$ \\
\hline
\end{tabular}

4.3 Ranking of the decision of risk level based on the VIKOR method of trapezoidal vague number

According to the decision steps of the VIKOR method, the aggregation operation of the trapezoidal vague evaluation value given by experts was performed. The results are shown in Table 8. According to Formula (13), the positive and negative ideal solutions are $\tilde{\alpha}^{+}$and $\tilde{\alpha}^{-}$, which are respectively defined as follows:

$\tilde{\alpha}^{+}=\{([5.084,7.442,9.652,12.29] ; 0.7801,0.7882),([4.005,8.453$, $10.88,13.66] ; 0.7967 .0 .765),([5.69,8.05,10.65,13.95] ; 0.812$, $0.7934),([4.65,8.251,11.99,17.54] ; 0.734,0.823),([5.45,8.62$, $10.61,13.192] ; 0.71,0.80)\}$

$$
\begin{aligned}
\tilde{\alpha}^{-}= & \{([3.2489,5.194,8.126,10.77] ; 0.592,0.643),([2.769,5.873, \\
& 8.694,13.075] ; 0.545,0.638),([3.5212,6.965,9.98,12.904] ; \\
& 0.7149,0.4888),([3.9715,6.9891,9.984,13.577] ; 0.55,0.69), \\
& ([2.56,4.55,9.07,11.81] ; 0.447,0.603)\}
\end{aligned}
$$

Then, Formula (14) and Formula (15) were used to calculate individual regret values of group benefit $S_{i}$ and $R_{i} i=(1,2, \cdots, m)$ for each risk level. The values were as follows: $S_{1}=1.3334, S_{2}=12.574, S_{3}=1.4727, S_{4}=1.8323$, $R_{1}=0.5026, R_{2}=3.2911, R_{3}=0.575$, and $R_{4}=0.678$.

Next, Formula (16) was used to determine the benefit ratio $Q_{i}$ of each risk level, where $\delta$ is the decision-making mechanism coefficient and $\delta=0.5$. The calculated $Q_{i}$ values are $Q_{1}=0, Q_{2}=1, Q_{3}=0.02$, and $Q_{4}=0.05$.

The benefit value $S_{i}$, individual regret value $R_{i}$, and benefit ratio $Q_{i}$ are in the order of $S_{1}<S_{3}<S_{4}<S_{2}$, $R_{1}<R_{3}<R_{4}<R_{2}$ and $Q_{1}<Q_{3}<Q_{4}<Q_{2}$. According to the above principle, the smaller the value, the better it is. The four risk levels are ranked as $Y_{2}<Y_{4}<Y_{3}<Y_{1}$, which means that the construction risk level of the long inclined shaft (No. 2 experimental well) in the Taigemiao mining area of the Shenhua Group is classified as Level 1.

\subsection{Comparative analysis of various decision-making methods}

Past studies [31, 32] have presented two decision models, and the comparative analyses are as follows:

1) Based on the calculation method of the score value in [31], there are $s\left(Y_{1}\right)=2.103, s\left(Y_{2}\right)=-0.326, s\left(Y_{3}\right)=0.681$, and $s\left(Y_{4}\right)=0.517$. According to the principle that the higher the score $s(Y)$ is, the better it is, the order is $Y_{2}<Y_{4}<Y_{3}<Y_{1}$. Then, based on the calculation method of utility value in [32], $m\left(Y_{1}\right)=0.513, m\left(Y_{2}\right)=0.241, \quad m\left(Y_{3}\right)=0.394$ and $m\left(Y_{4}\right)=0.415$ are available. According to the principle that the greater the utility value $m(Y)$, the better it is, we know that the order is $Y_{2}<Y_{4}<Y_{3}<Y_{1}$. Then, based on the method proposed in this study, the principle of lower benefit ratio $Q(Y)$ is better, because of $Q_{1}=0, Q_{2}=1, Q_{3}=0.02$, and $Q_{4}=0.05$. We know that the order is $Y_{2}<Y_{4}<Y_{3}<Y_{1}$. The comparison curves of the three methods are shown in Figure 1.

2) It can be seen that, among the three decision methods, the optimal and the worst risk evaluation grades of TBM construction in the long inclined shaft in a coal mine have not changed, that is, the risk evaluation grade $Y_{1}$ is the largest, and the risk evaluation grade $Y_{2}$ is the smallest. Moreover, the ranking results obtained by the trapezoidal vague number score value method and the VIKOR method are the same. Therefore, from good to bad, the ranking of TBM construction risk evaluation grade of the long inclined shaft in a coal mine is $Y_{2}<Y_{4}<Y_{3}<Y_{1}$.

\section{Conclusion}

The TBM construction of a long inclined shaft in a coal mine is a complex uncertain system. Such a complexity is mainly reflected in the numerous influencing factors and strong variability in data monitoring. Uncertainty is reflected in the selection of index value as a fuzzy interval value, rather than the determined real value. The uncertainty runs through the whole process of coal mine construction risk prediction. In order to solve this problem, a construction risk 
prediction method based on the trapezoidal vague set was proposed in this study, and the effectiveness of the method was verified through engineering examples. The main conclusions are presented below:

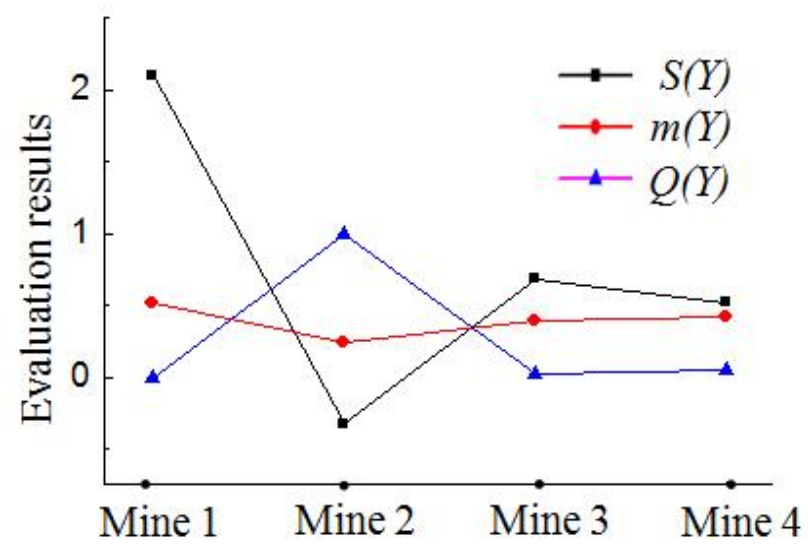

Fig.1. The evaluation results contrast curves of the three models

(1) The impact ground pressure factor, collapse factor, rock burst factor, and TBM jam factor were integrated into the secondary index concentration, which further enriched the evaluation index system of the TBM construction risk analysis of a long inclined shaft in a coal mine.

(2) The risk prediction method of coal mine construction based on the trapezoidal vague number can comprehensively describe and deal with the uncertainty in the monitoring information. The weighted arithmetic average operator is easy to program, which solves the problem of common methods finding it difficult to assemble fuzzy information quickly and effectively.

(3) The prediction results of coal mine construction risk based on the VIKOR model are consistent with the actual construction safety situation. Some feasible and effective evaluation methods have been provided for coal mine managers.

The risk prediction model was studied based on the fuzziness of coal mine construction monitoring information. Compared with the traditional method, the risk prediction model proposed in this study can more accurately reflect the risk grade state of the TBM construction process in a long inclined shaft in a coal mine. However, the monitoring information is ambiguous and time-varying; thus, how to establish a risk monitoring system that can consider realtime updating of data is worthy of further study.

\section{Acknowledgment}

This work was supported by the Scientific Research Start-up fund of the High-level Talents Introduction Project of Sanming University (19YG03), the Education and Scientific Research Project for Young and Middle-aged Teachers of the Fujian Province Education Department (JAT190690, B201903), Natural Science Foundation of Fujian Province of China(2020J01391, 2020J01384), and by the Joint Guiding Project of the Heilongjiang Natural Science Foundation (LH2019E085).

This is an Open Access article distributed under the terms of the Creative Commons Attribution License

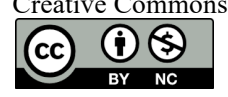

\section{References}

1. Li, X, P., Li, Y, A., "Research on risk assessment system for water inrush in the karst tunnel construction based on GIS: Case study on the diversion tunnel groups of the Jinping II Hydropower Station". Tunneling \& Underground Space Technology, 40(2), 2014, pp.182191.

2. Komal., "Fuzzy fault tree analysis for patient safety risk modeling in healthcare under uncertainty". Applied Soft Computing,37(12), 2015, pp.942-951.

3. Waddingham, E., Mt-Isa, S., Nixon, R., "A bayesian approach to probabilistic sensitivity analysis in structured benefit-risk assessment". Biometrical Journal, 58(1), 2016, pp.28-42.

4. Li, M.,Wang, H. T., "Risk assessment of gas explosion in coal mines based on fuzzy AHP and bayesian network". Process Safety and Environmental Protection, 135, 2020, pp.207-218.

5. Liu, P. D., Zhang X., "Approach to multi-attributes decision making with intuitionistic linguistic information based on dempster-shafer evidence theory". IEEE Access, 6(1), 2018, pp.52969-52981.

6. Yu, G. F., Fei W, Li D F., "A compromise-typed variable weight decision method for hybrid multi-attribute decision making". IEEE Transactions on Fuzzy Systems, 27(5), 2019, pp.861-872.

7. Paternesi, A., Schweiger, H. F., Scarpelli G., "Numerical analyses of stability and deformation behavior of reinforced and unreinforced tunnel faces". Computers and Geotechnics, 88, 2017, pp.256-266.

8. Nut, M., Tamara, A. B., Abdul-Hamid, S., "Probabilistic analysis and design of strip foundations resting on rocks obeying Hoek-Brown failure criterion". International Journal of Rock Mechanics \& Mining Sciences, 49(1), 2012, pp.45-58.

9. Wang, G. F., Xu, Y. X., Ren, H. W., "Intelligent and ecological coal mining as well as clean utilization technology in China: Review and prospects". International Journal of Mining Science and Technology, 29(2), 2019, pp.161-169.

10. Sideratos, G., Hatziargyriou, N. D., "An advanced statistical method for wind power forecasting”. IEEE Transactions on Power Systems, 22(1), 2007, pp.258-265.
11. Alexandre, G., Gerard, F., Regine, L., "Nonlinear acoustic echo cancellation based on volterra filters". IEEE Transactions on Speech and Audio Processing, 11(6), 2003, pp.672- 683.

12. Mollon, G., Dias, D., Soubra, A. H., "Range of the safe retaining pressures of a pressurized tunnel face by a probabilistic approach". Journal of Geotechnical and Geoenvironnmental Engineering, 139(11), 2013, pp.1954-1967.

13. Bajeel, P. N., Kumar, M., "Reliability test plan for a series system with variable failure rates". International Journal of Quality \& Reliability Management, 34(6), 2017, pp.849-861.

14. Aladejare, A. E., Wang, Y., "Influence of rock property correlation on reliability analysis of rock slope stability: from property characterization to reliability analysis". Geoscience Frontiers, 9(6), 2018, pp.1639-1648.

15. Wang, X. T., Li, S. C., Xu, Z. H., “An interval risk assessment method and management of water inflow and inrush in course of karst tunnel excavation". Tunnelling and Underground Space Technology, Retrieved from , 2019-10-30/2020-06-28.

16. Martin, J., Alipour, A., Sarkar, P., "Fragility surfaces for multihazard analysis of suspension bridges under earthquakes and microbursts". Engineering Structures, 197, 2019, pp.1-13.

17. Yang, B. B., Sui, W. H., Duan, L. H., "Risk Assessment of Water Inrush in an Underground Coal Mine Based on GIS and Fuzzy Set Theory". Mine Water and the Environment. 36(4), 2017 pp.617627.

18. Wang, Y., Lu, C. J., Zuo, C. P., "Coal mine safety production forewarning based on improved BP neural network". International Journal of Mining Science and Technology, 25(2), 2015, pp.319324.

19. Wang, Q. X., Wang, H., Qi, Z. Q., “An application of nonlinear fuzzy analytic hierarchy process in safety evaluation of coal mine". Safety Science, 86, 2016, pp.78-87.

20. Zhang, K., Dwayne, D., Zheng, W. B., "Prediction of karst for tunnelling using fuzzy assessment combined with geological 
investigations". Tunnelling and Underground Space Technology, 80, 2018, pp.64-77.

21. Sweta, B., Sutapa, P., Sanghamitra, D., "Fire monitoring in coal mines using wireless underground sensor network and interval type-2 fuzzy logic controller". International Journal of Coal Science \& Technology, 6(2), 2019, pp.274-285.

22. Deng, T. Q., Wang, X. F., "An object parameter approach to predicting unknown data in incomplete fuzzy soft sets". Applied Mathematical Model, 37(6), 2013, pp.4139- 4146.

23. Chen, X. G., Du, H., Yang, Y., "The Interval-valued triangular fuzzy soft set and Its method of dynamic decision making”. Journal of Applied Mathematics, Retrieved from https://doi.org/10.1155/2014/132806, 2014-01-22/2020-05-28.

24. Wang, Y. C., Chen, F., Yin, X., "Study on the risk assessment of water inrush in karst tunnels based on intuitionistic fuzzy theory". Geomatics Natural Hazards and Risk, 10(1), 2019, pp.1070-1083.

25. Wu, J., Sun, Q., Fujita, H., "An attitudinal consensus degree to control feedback mechanism in group decision making with different adjustment cost”. Knowledge-Based Systems, 164(15), 2019, pp.265-273.

26. Chen, X. G., Yang, Y., Bian, X. F., "Improvement of the method to determine weight based on the intuitionistic fuzzy entropy". International Journal of Hybrid Information Technology, 9(8), 2016, pp.377-386.
27. Xia, M. M., Xu, Z. S., "Entropy/cross entropy-based group decision making under intuitionistic fuzzy environment". Information Fusion, 13(1), 2012, pp.31-47.

28. Liu, P. D., Wang, P., "Some q-rung orthopair fuzzy aggregation operators and their applications to multiple-Attribute decision making". International Journal of Intelligent Systems, 33(2), 2018, pp.259-280.

29. Galindo, H., Gallardo, J. M., Jiménez-Losada, A., “A real Shapley value for cooperative games with fuzzy characteristic function". Fuzzy Sets and Systems, Retrieved from https://doi.org/10.1016/j.fss, 2020-04-19/2020-05-28.

30. Khaleed, A., Nasruddin, H., "Vgue soft multiset theory". International Journal of Pure and Applied Mathematics, 93(4), 2014, pp.511-523.

31. Kuang, T. L., "Trapezoidal-valued fuzzy soft sets and its applications". Advances in Information Sciences and Service Sciences, 4(15), 2012, pp.310-316.

32. Chen, X. G., Yu, G. F., Wu, J., "A minimum trust discount coefficient model for incomplete information in group decision making with intuitionistic fuzzy soft set". International Journal of Fuzzy Systems. Retrieved from https://doi.org/10.1007/s40815-02000811-2, 2020-03-21/2020-05-28. 